\title{
ELECTRO-MECHANICAL MODELLING AND LOAD SWAY CONTROL OF GANTRY CRANES
}

\author{
النمذجة اللاخطية و التحكم في تمايل الحمل بالأوناش العملاقة
}

\author{
F.S. Al-Fares ${ }^{* *}$, T. G. A. Al-Fiqi ${ }^{*}$ H.R. Al-Mubarak*** and M.S. Al-Ajmi** \\ - Prof., Production Eng. Depl. Faculty of Eng. Ain Shams Universily, Cairo, Egyph, and Now with the Depl. \\ of Prod. Tech., College of Tech. Studies, Authority of Applied Edvcation, Kuwail, I/qi@yahooco uk. \\ ** Assistant Prof \\ *** Teacher; \\ Dept. of I'roduction Technolegy, Conllege of Tech. Studies, Authorily of Applied Education. Kuwail
}

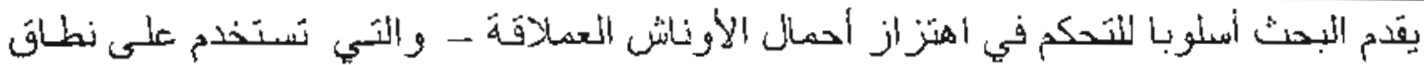

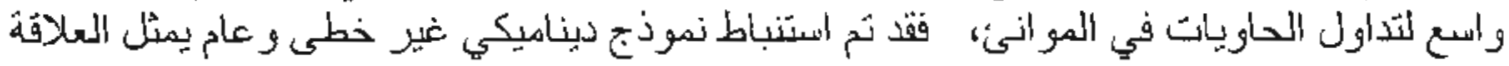

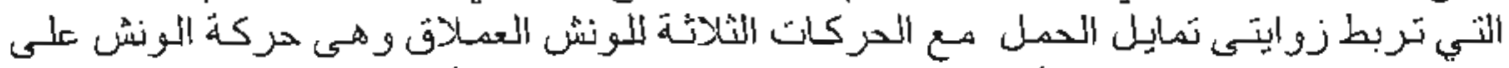

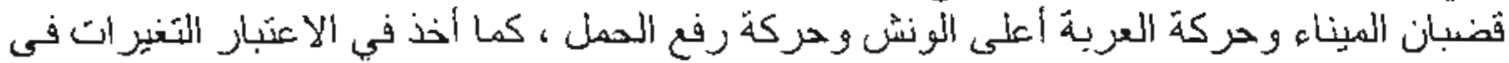

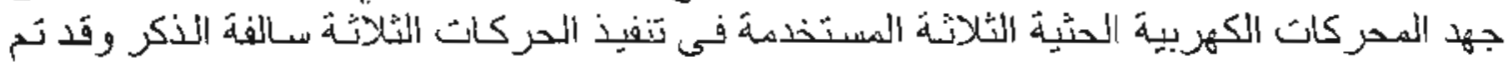

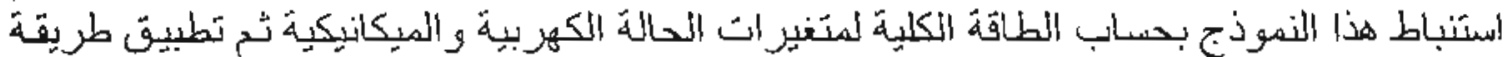

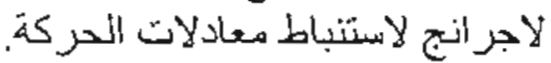

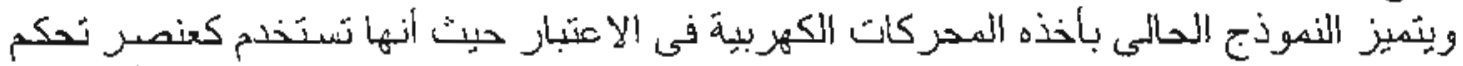

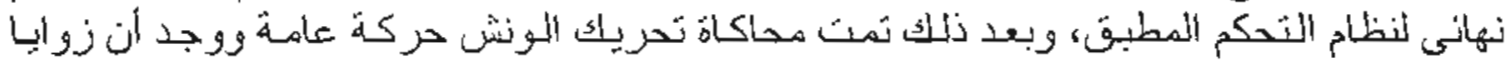

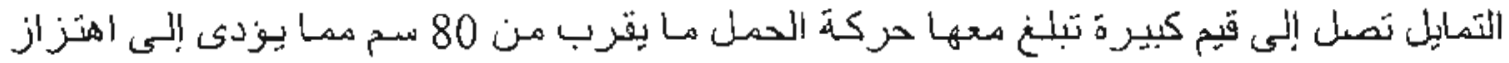

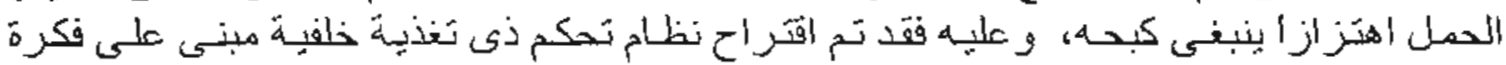

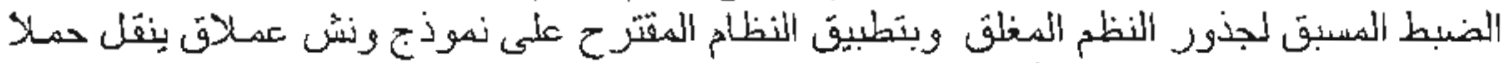

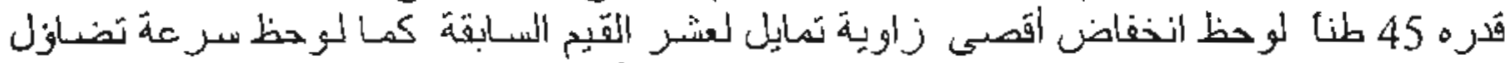

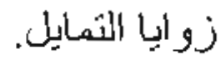

\section{ABSTRACT}

In this work, a nonlinear model representing the dynamics of the container sway of a gantry crane is derived. The dynamics of the induction motors are also taken into consideration in addition to the simultaneous iravelling, trolley and hoisting motions. The data of an actual 45-ton gantry crane is used to simulate the behavior of the container under an actual transportation plan. A simulation example is then presented to illustrate the uncontrolled system response. The container sway is found to be high such that a control scheme has to be implemented to suppress the load sway. A feedback control scheme is developed to suppress load sway. The feedback control matrix is chosen such that the poles of the closed-loop system are arbitrary assigned. The control scheme is applied and the simulation results illustrate the effectiveness of the proposed scheme.

Keywords: Gantry Cranes, Dynamic Modelling, Electro-Mechanical Systems, Anti-Sway Control.

Accepted December 30, 2007 


\section{59 F.S. Al-Fares, T.G.A. Al-fiqi, H.R. Al-Mubarak and M.S. Al-Ajmi}

\section{INTRODUCTION}

Gantry cranes are widely used in ports, and are installed at quayside to handle heavy containers. The necessity to increase the travelling, irolley and hoisting speed generaily induces undesirable sway of the container, and serious damage could occur during load Iransportation. Therefore, a satisfactory control scheme, based on an accurate dynamical model, is desirable to suppress load sway.

In the last decades, several investigators considered the modelling and control of gantry cranes as a nonliear control poblem [14]. Most of existing approaches consist of a two-stage procedure: off-line trajectory/path planning, carried out in accordance with proper optimisation criteria, and on-line tracking by traditional coniroliers. Optimal control techniques have been widely used to address the path pianning problem. Specific paths minimising traveling time, energy consumption or proper performance indexes linked to the swing angle and its derivative have been proposed in the literature. Nevertheless, due to model uncertainties and many other implementation faclors, it often happens that the actual crane behavior significantly differs from the "optimal", desired, one. The usual goal is to achieve zero swing only at the end of the transport, and a two-stage control structure is often used: a "tracking" controller during the load transfer, and a "stabilizing" one to be switched on when a suitable vicinity of the destination point is achieved. Barmeshwar et al. [5] proposed a norlinear control strategy for the trolly crane system using Lyapunov method without considering the sway angle dynamics in the stability analysis. Fang et al. [6] designed a proportional derivative (PD) controller to regulate the overhead crane system to the desired position with natural damping of sway oscillation. Liu et al. [7] developed a fuzzy logic control with sliding mode control for an overhead crane syslem. Fang et al. [8] developed a nonlinear coupling control law to stabilize a 3-DOF overhead crane by using Lasalle invariance theorem. However the parameters must be known in advance. Burg et al. [9] used the variable transformation method to regulate the crane system. d'Andrea-Novel and Boustany [10] proposed an adaptive feedback linearization method for mechanical systems of the overhead crane type. Karkoub and Zribi [11] used the pssivity property of mechanical system in the design of the nonlinear control of overhead crane. lshide et al. [12]construct a fuzzy back-propagation neural network based control. However their results have shown that the speed of the trolly is large at the desired destination. Yu et al. [13] developed a nonliear tracking for load position and velocily. However the results are shown for sway angle dynamics much faster than the cart motion dynamics. Another approch is developed by Kiss et al. [14] in which an output feedback PD controller is used to slabilize a nonlinear crane system. Benhidjeb and Gissinger [15] compared a fuzzy logic control system with Linear Quadratic Guassian control (LQG) for an overhead crane.Yi et al. [16] developed a fuzzy controller for anti-swing and position control for an overhead crane based on Single Input Rule Modules (SIRMs) dynamically connected fuzzy interference model. M. ElRaheb studied the effect the cable fiexibility on the load. It is found that cable flexibility has negligible effect on tlexural response. However, the above work neglected the dynamics of the driving electrical motors, which could lead to considerable errors. Also, it is convenient to use the inputs of the driving motors as control variables.

In this work, a nonlinear dynamic model of a gantry crane is derived. The dynamics of the induction motors as well as the simultaneous travel, transwerse and hoisting motions are taken into consideration. The derived model is 
then linearized about the normal operating conditions, and a simulation example is then presented to illustrate the container sway under an actual transportation plan. $\mathrm{lt}$ is found that the sway is relatively ligh. Therefore a control scheme is proposed to suppress the container sway. The torques of the driving crane motors are adjusted by feeding back the sway angles and their derivatives. The feedback control matrix is chosen such that the poles of the closed-loop system are arbitrary assigned. The control scheme is applied and the simulation results illustrate the effectiveness of the proposed scheme.

\section{DYNAMICAL MODELLING}

The dynamical model of the gantry crane shown in Fig. Ib will be derived. The crane travels parallel to the quayside on the railway while the trolley moves in the transverse direction carying the container. The hoisting of the container takes place during transportation. The container, whicls can be assumed as a suspended load from point $O$, will be assumed as a rigid body. Crane motors are induction motors for their simplicity and reliability. In the following analysis, the travel, hoisting and trolley motors will be modelled using the $d-q$ technique [20]. The kinetic and potential energies of the compound elecirocmechanical system will be obtained, and the dynamic equations associated with the generalized coordinates will be derived, using the Lagrangian approach. This will be derived and presented in the following subsections.

\subsection{Lagrangian Function of the SYstem}

The coordinates of the container $x_{\mathrm{r}}, y_{\mathrm{s}}$ and $z_{\mathrm{s}}$ can be expressed in terms of the coordinates of the crane, " $11, v$ and $w$ ", the container sway angle, $\theta_{1}$ and $\theta_{2}$, and the length of the wire " $\%$ " as follows;

$$
\begin{aligned}
& x_{\mathrm{r}}=u+l \sin \theta_{1} \sin \theta_{2}, \\
& y_{c}=v+l \sin \theta_{1} \cos \theta_{2}, \text { and } \\
& z_{c}=w+l \cos \theta_{1}
\end{aligned}
$$

The kinetic energy of the mechanical system represents the kinetic energy of the suspended container of mass $M$, the trolley and the travelling gantry crane. The nomenclature can be found at the end of the paper, and the kinetic energy can be written as follows;

$$
\begin{aligned}
K . E_{r_{m}} & =\frac{1}{2} M\left(\dot{x}_{c}^{2}+\dot{y}_{c}^{2}+\dot{z}_{c}^{2}\right)+\frac{1}{2} m_{1} \dot{u}^{2} \\
& +\frac{1}{2} I_{a} \dot{\theta}_{2}^{2} \cos ^{2} \theta_{1}+\frac{1}{2} m_{2}\left(u^{2}+\dot{v}^{2}\right) \\
& +\frac{1}{2} I_{r}\left(\theta_{1}^{2}+\dot{\theta}_{2}^{2} \sin ^{2} \theta_{1}\right)+\frac{1}{2} I_{1}\left(\frac{\dot{u}}{r_{1}}\right)^{2} \\
& +\frac{1}{2} I_{2}\left(\frac{\dot{v}}{r_{2}}\right)^{2}+\frac{1}{2} I_{3}\left(\frac{j}{r_{3}}\right)^{2}
\end{aligned}
$$

The potential energy of the mechanical subsystem represents the potential energy of the suspended container, and the stored energy in the wire ropes of stiffness $Q$. The following expression of the potential energy can be obtained;

$P . E_{m}=\frac{1}{2} Q \phi^{2}-M g / \cos \theta_{1}$

The equivalent kinetic energy of the crane induction motors; namely the travelling motor " $r$ ", trolley motor " $r$ " and the hoisting motor " $h$ " can be expressed in the following equations;

$$
\begin{aligned}
& K . E_{s}=\frac{1}{2}\left[\begin{array}{c}
L_{t r} I_{t s} I_{t s}^{\prime}+L_{t r} I_{t r} I_{t r}^{\prime} \\
+L_{t m}\left(I_{s s} I_{t r}^{\prime}+I_{s s}^{\prime} I_{t r}\right)
\end{array}\right] \\
& K . E_{v_{r}}=\frac{1}{2}\left[\begin{array}{l}
L_{r r} I_{r} I_{r}^{*}+L_{r r} I_{r r} I_{r r}^{*} \\
+L_{r m}\left(I_{r r} I_{r r}^{*}+I_{r}^{*} I_{r r}\right)
\end{array}\right] \\
& K E_{\cdot h}=\frac{1}{2}\left[\begin{array}{l}
L_{h s} I_{h r} I_{h r}^{*}+L_{k r} I_{h r} I_{h r}^{*} \\
+L_{h r r}\left(I_{h r} I_{h r}^{*}+I_{h r}^{*} I_{h r}\right)
\end{array}\right]
\end{aligned}
$$

Now, the kinetic and potential energy of the system can be expressed in the eqs. (1-5), and the Lagrangian function can be obtained as follows:

$L=K \cdot E_{m}+K E_{. t}+K E_{.,}+K \cdot E_{, p}-P \cdot E_{m}$

which can be written as; 


$$
\begin{aligned}
& L=\frac{1}{2}\left[L_{t r} I_{t r} I_{t r}^{*}+L_{t r} I_{t r} I_{t r}^{*}+L_{r s t}\left(I_{t r} I_{t r}^{*}+I_{t r}^{*} I_{t r}\right)\right] \\
& +\frac{1}{2} m_{1} \dot{u}^{2}-\frac{1}{2} Q \phi^{2}+M g l \cos \theta_{1} \\
& +\frac{1}{2}\left[L_{r r} I_{n r} I_{r s}^{*}+L_{r r} I_{r r} I_{r r}^{*}+L_{r m}\left(I_{n} I_{r r}^{*}+I_{n}^{*} J_{r r}\right)\right] \\
& +\frac{1}{2} M\left(\dot{x}_{c}^{2}+\dot{y}_{c}^{2}+\dot{z}_{c}^{2}\right)+\frac{1}{2} m_{2}\left(\dot{u}^{2}+\dot{v}^{2}\right) \\
& +\frac{1}{2}\left[L_{h s} I_{h r} I_{h s}^{*}+L_{h r} I_{h r} I_{h r}^{*}+L_{h r s}\left(I_{h s} I_{h r}^{*}+I_{h s}^{*} I_{h r}\right)\right] \\
& +\frac{1}{2} I_{a} \dot{\theta}_{2}^{2} \cos ^{2} \theta_{1}+\frac{1}{2} I_{1}\left(\dot{\theta}_{1}^{2}+\dot{\theta}_{2}^{2} \sin ^{2} \theta_{1}\right) \\
& +\frac{1}{2} I_{1}\left(\frac{\dot{u}}{r_{1}}\right)^{2}+\frac{1}{2} I_{2}\left(\frac{\dot{v}}{r_{2}}\right)^{2}+\frac{1}{2} I_{3}\left(\frac{i}{r_{3}}\right)^{2}
\end{aligned}
$$

The above Lagrangian equation will be used to derive the dynamic differential equation of the system.

\subsection{Modelling of the Electrical Sub-Svstem}

In the following, the Lagrangian function derived in eq. (6) will be differentiated to obtain the dynamic equation governing the transients of one of the crane induction motors. These motors are the travelling molor " $r$ " trolley motor " $c$ ", and the hoisting motor " $h$ " and the subscript " $i$ " will refer to one of them. First, differentiate with respect to the stator current, the following equation can be obtained;

$\frac{d L}{d J_{i s}}=L_{w s} I_{t s}+L_{r m} I_{t s}=W_{t s}$

The right hand side of eq. (7) is the stator flux. The above equation can be differentiated with respect to time to obtain the following equation;

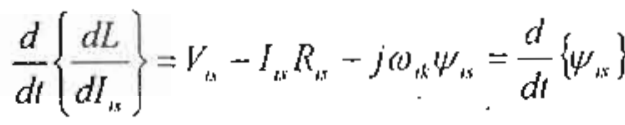

Regarding the rotor side, the above steps can be repealed to obtain the following equations;

$$
\frac{d L}{d I_{s r}}=L_{t r} I_{t r}+L_{i n t} I_{r r}=\psi_{t r}
$$

$$
\begin{aligned}
\frac{d}{d l}\left\{\frac{d L}{d I_{t r}}\right\} & =V_{r r}-I_{r r} R_{u r} \\
& -j\left(\omega_{r k}-\omega_{1}\right) \psi_{t r}=\frac{d}{d t}\left\{\psi_{u t}\right\}
\end{aligned}
$$

Equation (8) and eq. (10) represent the state equation of the ith motor, where the flux vectors are considered as states. However, it is convenient to use the primitive machine or the direct-quadrature phase quantities, $d-q$ representation. In the following, the above motor vector quantities are represented by its d-q components;

$$
\begin{aligned}
& \psi_{s s}=\psi_{s d}+j \psi_{s w y} \quad, \quad \psi_{s}=\psi \psi_{r r d}+j \psi_{s u}
\end{aligned}
$$

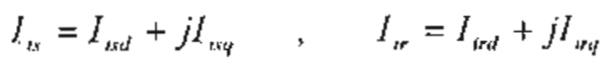

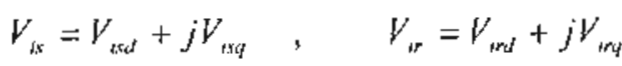

It is to be noted that the voltage on the rotor is zero; i.e. $V_{t r}=0$

Substituting the above relationships into eq. (8) and eq. (10), the following state equations can be written;

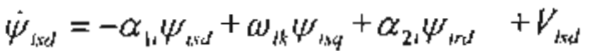

$$
\begin{aligned}
& \dot{\psi}_{i x q}=-\omega_{1,} \psi_{s u d}-\alpha_{i k} \psi_{s q q}+\alpha_{2 i} \psi_{i r q}+V_{s i d}
\end{aligned}
$$

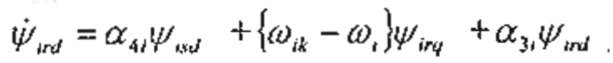

$$
\begin{aligned}
& \dot{\psi}_{\text {rrq }}=-\left\{\omega_{1 k}-\omega_{i}\right\} \psi_{\text {sid }}+\alpha_{4 r} \psi_{i r y}+\alpha_{3,} \psi_{\text {rrd }}
\end{aligned}
$$

The above equations represent the state equations of the ith motor, and can be written in matrix form as follows;

$$
\dot{X}_{1}=G_{1} X_{1}+V_{i} \quad, \quad i=r, l, h
$$

where; $X_{i}^{7}=\left[\begin{array}{llll}\psi_{t r i t} & \psi_{\mathrm{suq}} & \psi_{t r i t} & \psi_{\mathrm{rry}}\end{array}\right]$,

$$
V_{1}^{T}=\left[\begin{array}{llll}
V_{u d} & V_{\mathrm{sq}} & 0 & 0
\end{array}\right] \text {, and }
$$

$G_{r}=\left[\begin{array}{cccc}-\alpha_{1 r} & \omega_{r k} & \alpha_{2 t} & 0 \\ -\omega_{1 k} & -\alpha_{1 t} & 0 & \alpha_{2 t} \\ \alpha_{4 t} & \left(\omega_{1 k}-\omega_{1}\right) & -\alpha_{3 t} & 0 \\ -\left(\omega_{r k}-\omega_{r}\right) & \alpha_{4 r} & 0 & -\alpha_{3 k}\end{array}\right]$ 
The dynamics of the rotor and the motor torgue are included in the following fifth equation;

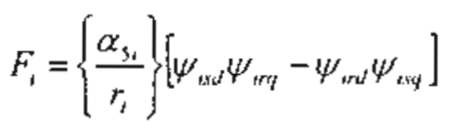

and if linearized;

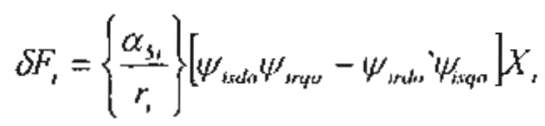

or in vector notation;

$$
\delta F_{1}=E, X_{1} \quad, \quad i=r, t, h
$$

\subsection{Modelling of the Mechanical sub-System}

Lagrange's equation will be used to obtain the differential equations associated with the generalized coordinates. Five generalized coordinates are associated with the mechanical side of the compound electromechanical system, while twelve coordinates are associated with the electrical part. The details of the state equations of the induction motors are given in section 2.2, while the derivation of the state equations for the mechanical side are given in the present section. The following five equations are associated with the mechanical generalized coordinates;

$$
\begin{aligned}
& A_{11} \ddot{u}+A_{13} \ddot{l}+A_{14} \ddot{\theta}_{1}+C_{133} i \dot{\theta}_{2}+C_{134} \dot{\theta} \dot{\theta}_{1} \\
& +C_{145} \dot{\theta}_{1} \dot{\theta}_{2}+C_{155} \dot{\theta}_{2}^{2}+C_{144} \dot{\theta}_{1}{ }^{2}+A_{15} \ddot{\theta}_{2}=F_{r} \\
& A_{22} \ddot{v}+A_{24} \ddot{\theta}_{1}+A_{23} \ddot{\theta}_{2}+C_{23} j \dot{\theta}_{2}+C_{234} i \dot{\theta}_{1} \\
& +C_{24 s} \dot{\theta}_{1} \dot{\theta}_{2}+C_{2 s s} \dot{\theta}_{2}^{2}+C_{2 \varsigma 4} \dot{\theta}_{1}{ }^{2}+A_{23} \ddot{l}=F_{\text {I }} \\
& A_{31} \ddot{i}+A_{32} \ddot{v}+A_{33} \ddot{l}+C_{335} \dot{\theta}_{2}^{2} \\
& +C_{144} \dot{\theta}_{1}^{2}=M g-F_{\text {h }} \\
& A_{41} \ddot{u}+A_{42} \ddot{v}+A_{44} \ddot{\theta}_{1}+C_{434} i \dot{\theta}_{1} \\
& +C_{45 s} \dot{\theta}_{2}^{2}+D_{44}=0 \\
& A_{31} \ddot{u}+A_{52} \ddot{v}+A_{3 y} \ddot{\theta}_{2}+C_{33} i \dot{\theta}_{2} \\
& +C_{45} \dot{\theta}_{2} \dot{\theta}_{1}+D_{35} \theta_{2}=-Q \phi
\end{aligned}
$$

where " $A_{i t}$ " represents the elements of the effective inertia matrix " $A$ ", which can be found in Appendix A. On the other side, the element " $C_{p k}$ " represents the coefficient of the dynamic force (centripetal or coriolis) at coordinate " $i$ "due to the velocities at " $j$ " and " $k$ ". These elements can be obtained from the $C(q, \dot{q})$, which can be found also in Appendix $A$. Regarding the gravity loading matrix $D$, every diagonal element " $D_{v}$ " represents the gravity loading at joint " $i$ ". All the elements of this matrix are zero except " $D_{44}=M g l \sin \theta_{1} "$. The above equations can be written as follows;

$$
A(q) \ddot{q}+C(q, \dot{q})+D(q)=F
$$

\section{SYSTEM SIMULATION}

Crane accelerations $\ddot{u}, \ddot{v}$ and $\ddot{l}$ are decided according to the transportation plan. Therefore, the motor inputs can be calculated by using the nonlinear electromechanical model. The above equations will be used to simulate the response of the considered crane for a specified transportation plan. The proposed plan is to move the gantry with a constant acceleration of $0.3 \mathrm{~m} / \mathrm{sec}^{2}$, and the trolley with a constant acceleration of 0.15 $\mathrm{m} / \mathrm{sec}^{2}$ for ten seconds. Then, the gantry and trolley moves with a constant velocity for another ten seconds. Finally, the gantry moves with a constant deceleration of 0.3 $\mathrm{m} / \mathrm{sec}^{2}$, and the trolley moves with a constant deceleration of $0.15 \mathrm{~m} / \mathrm{sec}^{2}$ for anoth ten seconds. The proposed plan is illustrated on Fig.2-3, and the response is presented on Fig. 4-5. Since in practice the suspension ropes are not wound while the crane is in motion for saftey considerations [4], no-hoisting of the load will be considered in this case. It is clear that the sway angles are increasing for both $\theta_{1}$ and $\theta_{2}$. It can be also noticed that the frequency of oscillation of $\theta_{1}$ is about $0.7 \mathrm{~Hz}$, which can be predicted from the system equation 33. From Fig.4-5, it can be noticed that $\theta_{1}$ reaches 0.035 radians, and $\theta_{2}$ exceeds 0.26 radians, withist 30 seconds. This means that the contianer 


\section{63 F.S. Al-Fares, T.G.A. Al-fiqi, H.R. Al-Mubarak and M.S. Al-Ajmi}

load of 45 ton vibrates with an amplitude of $70 \mathrm{~cm}$, and rotates about its axis with an angle of about 16 degrees, within 30 seconds. This dangerous state indicates the necessity of applying a control scheme to suppress the load sway. This will be considered in the next section. The data of the considered gantry are presented in Table I.

\section{LINEARIZED MODEL}

The aim of the present work is to suppress the load sway angles $\theta_{1}$ and $\theta_{2}$ during the transportation period. In order to facilitate the analysis of the above system, and the application of feedback control scheme, it is recommended to linearize the above model about the nominal values of the states.

The nominal values of the states can be obtained by setting the dervitaves of the sway angles to zero. Therefore, the nominal states, $\theta_{10}$ and $\theta_{20}$, can be obtained as follows;

$\theta_{10}=\sqrt{\left(\ddot{u}_{o}^{2}+\ddot{v}_{o}^{2}\right) / g}$

$\theta_{20}=\tan ^{-1}\left(\ddot{u}_{o} / \ddot{v}_{o}\right)$

Therefore, the equations 18-22 of the mechanical subsystem, after neglecting the higher order terms and substituting for the forces from equations 15-17, can be written as follows;

$$
\begin{aligned}
& A_{11} \delta \ddot{z i}+A_{130} \ddot{\delta t}+A_{140} \delta \ddot{\theta}_{1}+A_{150} \delta \ddot{\theta} \\
& +C_{1350} i_{0} \delta \dot{\theta}_{2}+C_{1,140} i_{0} \delta \dot{\theta}_{1}=\delta F_{r}=E_{r} X \text { r } \\
& A_{22} \delta \ddot{N}+A_{210} \ddot{\delta}+A_{240} \delta \ddot{\theta}_{1}+A_{150} \delta \ddot{\theta}_{2} \\
& +C_{23 s 0} i_{0} \delta \dot{\theta}_{2}+C_{2340} i_{0} \delta \dot{\theta}_{1}=\delta F_{t}=E_{i} X_{1} \\
& A_{310} \delta \ddot{u}+A_{320} \delta \dot{v}+A_{130} \ddot{\delta l}=-\delta F_{h}=-E_{\mathrm{h}} X_{\mathrm{h}}(28) \\
& A_{410} \delta \ddot{z i}+A_{420} \delta \dot{\delta i}+A_{440} \delta \ddot{\theta}_{1}+C_{4340} l_{0} \delta \dot{\theta}_{1} \\
& +D_{440} \delta \theta_{1}=0 \\
& A_{510} \delta \ddot{u}+A_{520} \delta \ddot{i}+A_{550} \delta \ddot{\theta}_{2} \\
& +C_{5350} l_{0} \delta \dot{\theta}_{2}-Q \phi=0
\end{aligned}
$$

According to the assumption of small perturbations about the nominal values, the non-holonomic constraint; $\dot{\phi}-\dot{\theta}_{2} \cos \theta_{1}=0$, associated with this system can be reduced to be; $\delta \dot{\phi}=\delta \dot{\theta}_{2}$. Therefore, equation 30 can be rewritten as follows;

$$
\begin{aligned}
A_{310} \delta \ddot{\delta i}+A_{320} \delta \dot{\delta}+A_{350} \delta \ddot{\theta}_{2}+C_{3350} i_{0} \delta \dot{\theta}_{2} \\
+Q \delta \theta_{2}=0
\end{aligned}
$$

The crane accelerations $\ddot{u}, \ddot{v}$ and $l$ are decided according to the transportation plan. Therefore, the motor inputs can be calculated by using the electromechanical model. Now, the following vectors will be introduced;

$\Psi^{T}=[\delta u, \delta v, \delta l] \& \Theta^{T}=\left[\delta \theta_{1}, \delta \theta_{2}\right]$

where the first vector " $\Psi$ " represents the gantary motions which are decided according to the proposed transportation plan, and the second vector " $\Psi$ " represents the controlled states of the considered system. Now the system dynamic equation of motion can be written as follows;

$$
\begin{aligned}
& J \ddot{\Theta}+D \dot{\Theta}+K \Theta=B \ddot{\Psi} \\
& J_{1} \ddot{\Theta}+D_{1} \dot{\Theta}+B_{1} \ddot{\Psi}=\left[\begin{array}{ll}
E_{r} X_{r}, & E_{1} X_{l},
\end{array}-E_{h} X_{h}\right]^{T} \\
& J_{1} \ddot{\Theta}+D_{1} \dot{\Theta}+B_{1} \ddot{\Psi}=F_{l}
\end{aligned}
$$

The parameters of the matrices can be found in Appendix A.

\section{PROPOSED CONTROL SYSTEM}

The above linearized system wil] be used to apply a control scheme such that the load sway can be suppressed. The considered system will be shown to be controllable, before applying the control scheme. Equations 33-34 can be reduced to the following form;

$$
\begin{aligned}
& J \ddot{\Theta}+D \dot{\Theta}+K \Theta= \\
& \quad-B B_{1}^{-1} J_{1} \ddot{\Theta}-B B_{1}^{-1} D_{1} \dot{\Theta}+B B_{1}^{-1} F_{t}
\end{aligned}
$$

which can be rewritten as follows;

$$
\ddot{\Theta}+\xi \dot{\Theta}+\Omega \Theta=\beta F_{F}
$$

which has the following state space presentation; 
$\dot{X}=A X+B E$

where;

$\mathrm{A}=\left[\begin{array}{cc}0 & I \\ -\Omega & -\xi\end{array}\right], \mathrm{B}=\left[\begin{array}{l}0 \\ \beta\end{array}\right], \mathrm{X}=\left[\begin{array}{c}\Theta \\ \dot{\Theta}\end{array}\right], \mathrm{E}=\left[\begin{array}{c}0 \\ F_{E}\end{array}\right]$

The above system will be controllable if the following controllability matrix;

$\Delta=\left\lfloor\mathrm{B}, \mathrm{AB}, \mathrm{A}^{2} \mathrm{~B}, \mathrm{~A}^{3} \mathrm{~B}\right\rfloor$

has the full rark. If the matrix " $\mathrm{A}$ " is cyclic, a rank "f" feedback control scheme can be applied where the two input model is reduced to single input model. This control scheme fixes the ratio of the two inputs to the motors according to the following [18];

$$
\mathrm{E}=\mathrm{Z} X
$$

where "Z" is a vector of constants to be chosen such that the reduced system is controllable from the new single input " $\chi$ ". Now the system equation 36 can be written as follows;

$$
\dot{\mathrm{X}}=\mathrm{A} \mathrm{X}+(\mathrm{BZ}) \chi
$$

The feedback control will be carried out by assuming the control action to be;

$$
x=-\mathrm{K}^{T} \mathrm{X}
$$

where " $\mathrm{K}$ " is a gain vector to be chosen such that all eigenvalues of the feedback control system are assigned as desired. It will be now shown that the matrix " $A$ " is cyclic and then to select a vector " $Z$ " that ensures the controllability of the reduced system. The matrix "A" will be cyclic if the matrix "sI-A", " $s$ " is the Laplace transform variable, has one non-unity invariant polynomial [18]. The invariant polynomials of the of "sI-A" can be obrained as follows;

$$
\Lambda_{1}=\left\{\begin{array}{ll}
1 & i=1,2,3 \\
\sum_{k=0}^{4} \varepsilon_{k} s^{4-k} & i=4
\end{array}\right\}
$$

From the above equation, and the value of the system parameters, it is clear that last invariant polynomial is non-unity and consequently the matrix " $\mathrm{A}$ " is cyclic. The existence of a vector " $Z$ " which is capable to make the reduced system equation (38) controllable is ensured by the following Lemma (see [18] for the proof).

Lemma: If the multi-input system described by equation (36) is controllable and its system matrix " $A$ " is cyclic, then almost any vector " $Z$ " will make the reduced single-input system described by eq. (38) controllable, i.e. the controllability matrix of this system

$$
\Delta_{r}=\left[\mathrm{B} Z, \mathrm{ABZ}, \mathrm{A}^{2} \mathrm{~B} Z, \mathrm{~A}^{3} \mathrm{~B} Z\right]
$$

has the fult rank.

\subsection{Selection of Feedback Gain}

The characteristic polynomial of the system withoul feedback can be written as follows;

$s^{4}+\varepsilon_{1} s^{3}+\varepsilon_{2} s^{2}+\varepsilon_{3} s+\varepsilon_{4}=0$

If the desired eigenvalues of the system with feedback are assigned as $\lambda_{1}, \lambda_{2}, \lambda_{3}, \lambda_{4}$, the closed characteristic equation can be considered as follows;

$s^{4}+\mu_{1} s^{3}+\mu_{2} s^{2}+\mu_{3} s+\mu_{4}=0$

The feedback gain vector that yields the desired closed loop eigenvalues can be derived as follows [18];

$$
\mathrm{K}=\left[\Delta_{\mathrm{r}}^{i}\right]^{-1} \Gamma^{-1}(\mu-\varepsilon)
$$

where $\Gamma$ is lower triangular Teoplitz matrix with the first column $\left[1, \varepsilon_{1}, \varepsilon_{2}, \varepsilon_{3}\right]^{T} ; \Delta_{r}$ is the controllability matrix defined in eq. (4I), and the vectors $\mu$ and $\varepsilon$ are defined as follows;

$$
\mu=\left[\mu_{1}, \mu_{2}, \mu_{3}, \mu_{4}\right]^{T} \text { and } \varepsilon=\left[\varepsilon_{1}, \varepsilon_{2}, \varepsilon_{3}, \varepsilon_{4}\right]^{T}
$$

The above results have been implemented to suppress the sway of the load. The poles of 
the closed loop are placed at " $-0.25 \pm j 0.75$ " and at " $-4 \pm j 8$ ", for the first ten seconds and the last ten seconds. Regarding the second ten seconds, the poles are placed as " $0.25 \pm j 0.75 "$. Fig. 6 shows the block diagram of the control scheme. Simulations have been carried out to test the performance of the feedback control scheme as applied to the considered gantry crane. The results are presented in Fig. 7. It is clear that the sway angle $\delta \theta_{1}$ now exhibits a decaying vibration charateristics and a maximum sway angle now of $0.0025 \mathrm{rad}$ has been obtained and zero sway will be obtained. Regarding the sway angle $\delta \theta_{2}$, it is sufficient to force $\delta \theta_{1}$ to zero to suppress the sway of the contianer.

\section{CONCLUSIONS}

A nonlinear dynamical model of the electromechanical system of gantry crane has been developed 10 represent the load sway dynamics, using the Lagrangian approach. This dynamical model considers the induced sway of the suspended load due to the simultaneous motions of the gantry, trolley and the hoisting of the load. The dynamics of the induction motors are also taken into consideration in addition to the afromentioned motions. The considered sway is induced not only in the plane of motion, but also in the plane determined by the ropes and the vertical axis through the suspension point.

The derived model is adopted to simulate the response of an actual gantry crane. The data of a 45-ton gantry crane is used to simulate the behavior of the container under an actual transportation plan. A simulation example is then presented to illustrate the uncontrolled system response. The container sway is found to be high such that a control scheme has to be implemented to suppress the load sway.

A feedback control scheme is developed to suppress load sway. The lorques of the driving crane motors are adjusted by feeding back the sway angles and their derivatives. The feedback control matrix is chosen such that the poles of the closed-loop system are arbitrary assigned. The control scheme is applied and the simulation results illustrate the effectiveness of the proposed scheme.

\section{Acknowledgement}

The authors acknowledge and appreciate the financial support of the Public Authority for Applied Education and Training under the Research Grant TS-06-04.

\section{References}

[1] Sakawa, Y. and ShindoY.,"Optimal Control of Containcr Cranes", Automatica, Vol.18-3, pp 257-266, 1982.

[2] Butler, H., Honderd, G., Amerongen, J.V. "Model reference adaptive control of a gantry crane scale model", [EEE Control Systems, pp. 57-62, 1991.

[3] Kenison, $M$. and Singhose, $W$. "Iriput shaper design for double pendulum planar gantry cranes", IMECE Conf., pp $55-59,1999$.

[4] Kim, Y-S.; Hong, K-S.and Seung$\mathrm{Ki}$, "Anti-sway control of container cranes" Int. J. of Control, Automation and Systems v 2 n 4 p 435-449, 2004.

[5] Barmeshwar Vikramaditya, Rajesh Rajamani. Nonlinear control of a trolley crane system." American control conference, Chicago, IL. 2000. p. 1032-6.

[6] Fang $Y$, Zergeroglu E, Dixon WE, Dawson DM. Nonlinear coupling control laws for an overhead crane system." IEEE Int. Conf. on control applications, Mexico. 2001. p. 639-44.

[7] Liu D, Yi J, Zhoa D. Fuzzy tuning sliding mode control of transporting for an overhead crane." 2nd Int. Conf. on machine learning and cybernetics, $X i^{\prime} a n$, China. 2003. p. $2541-6$.

[8] Fang $Y$, Dixon WE, Dawson DM, Zergeroglu E. Nonlinear coupling control laws for a 3-DOF overhead crane system. IEEE international conference on decision and control, Orlando, FL, USA. 2001.

[9] Burg T, Dawson D, Rahn C, Rhodes $w$. Nonlinear control for an overhead crane via the saturating control approach of tcel. In: Proceedings of the IEEE international conference on robotics and automation, Minnesota, USA. 1996.

[10] d'Andrea-Novel B, Boustany F. Adaptive control for a class of mechanical systems using linearization and Lyapunov methods. A comparative study on the 
overhead crane example", IEEE Int. Conf. decision and control, UK. 1991. p. 120-5.

[11] Karkoub MA, Zribi M. Modelling and energy based nonlinear control of crane lifter. IEE Proc-Control Theory Appl 2002;149(3): 209-16.

[12] Ishide $T$, Uchida $H$, Miyakawa $S$. Application of a fuzzy neural network in the automation of crane system", 9 th fuzzy system symposium. 1991. p. 29-33.

[13] Yu J, Lewis FL, Huang $T$. Nonlinear feedback control of a gantry crane", American control conference, Washington, USA. 1995. p. 4310-5.

[14] Kiss B, Levine J, Mul lhaupt Ph. A simple output feedback PD controller for nonlinear cranes", 39th IEEE conf. on decision \& control, Australia. 2000.

[15] Benhidjeb A, Gissinger GL. Fuzzy control of an overhead crane per[ormance comparison with classic control", Control Eng Practice 1995;(12): 1687-96.
[16] Yi Jianqiâng, Yubazaki Naoyoshi, Hirota Kaoru. Anti-swing and positioning control of overhead traveling crane. Inform Sci 2003; 155:19-42.

[17] El-Raheb; M.; "Effect of cable flexibility on transient response of a beam-pendulum system"; J. of Sound and Vibration, V.307, Issues 3-5, 6 Nov. 2007, P. 834-848.

[18] Van De Vegte, "Feedback Conirol Systems" Prentice-Hall, 1986.

[19] "Technical data of 45-ton Gantry Crane", Tomen Corporation and Mitsubishi Heavy Ind. Lid., Japan, (2000).

[20] Begamudre, R.D., "ElectroMechanical Energy Conversion with Dynamics of Machines", Wiley 1988.

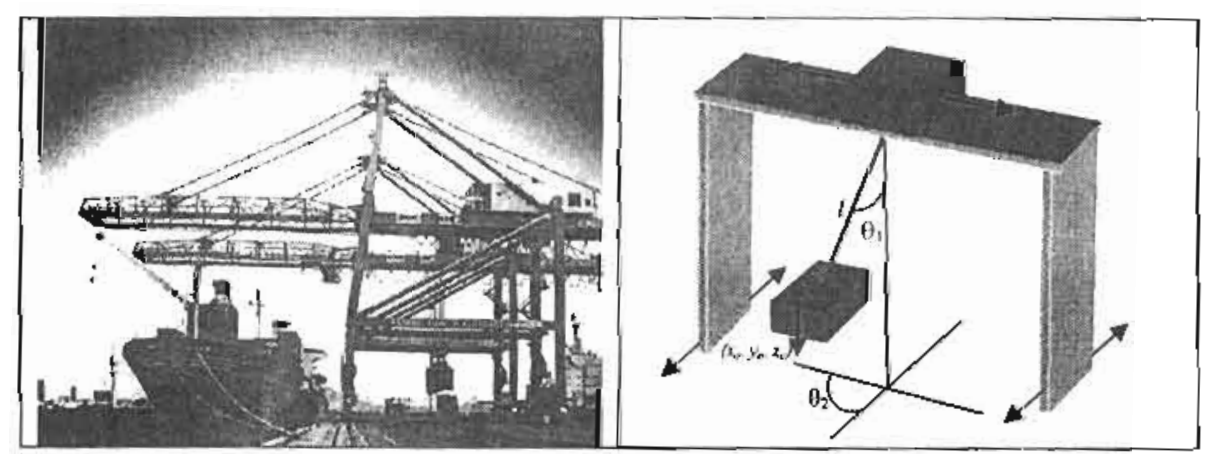

Fig 1. a) Acual gantry crane at Shungikh port in Kuwait, b) Free body diagram for gantry crane

Table 1. Data of the Considered Crane.

$m_{1}=1000000 \mathrm{~kg} ., \quad m_{2}=10000 \mathrm{~kg}, \quad M=45000 \mathrm{~kg} ., I_{\alpha}=40000 \mathrm{~kg} \cdot \mathrm{m}^{2}, \quad I_{a}=50000 \mathrm{~kg} \cdot \mathrm{m}^{2}, l=20 \mathrm{~m} . . Q=150$ N.ritrad, The data of the molors can be found in [19]. 


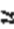

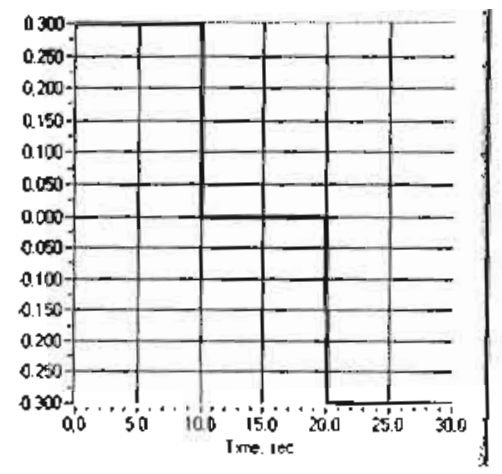

Fig. 2. Transportation PJan of "

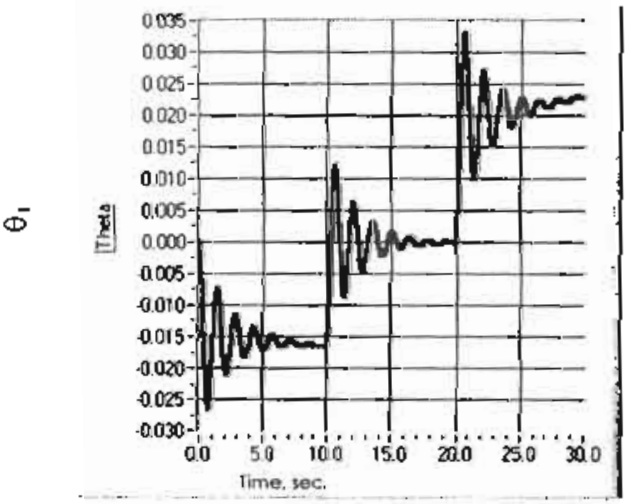

Fig. 4. Sway angle or $\theta_{i}$ without control

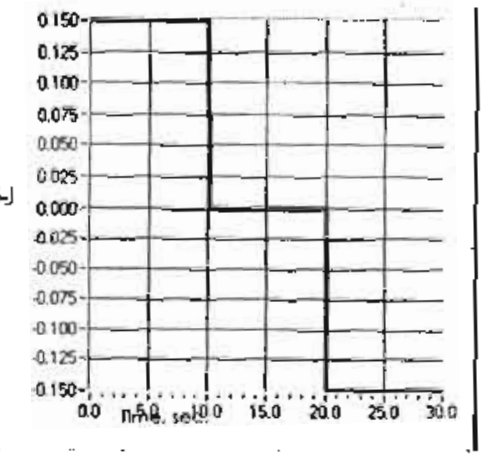

Fig. 3. Transportation Plan of $v$

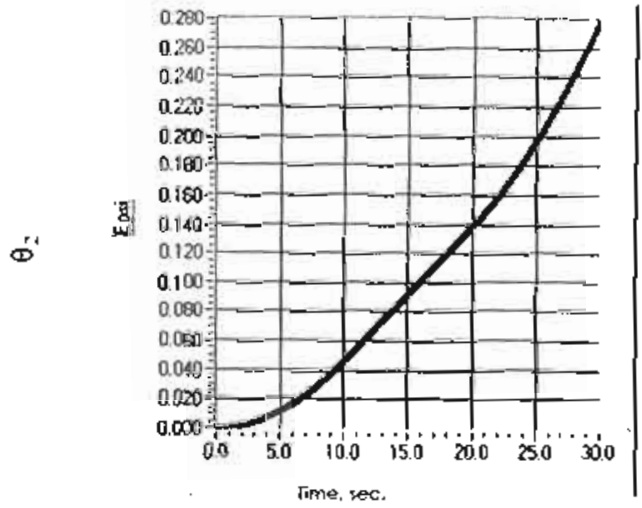

Fig. 5. Sway angle of $\theta_{2}$ withoul control.

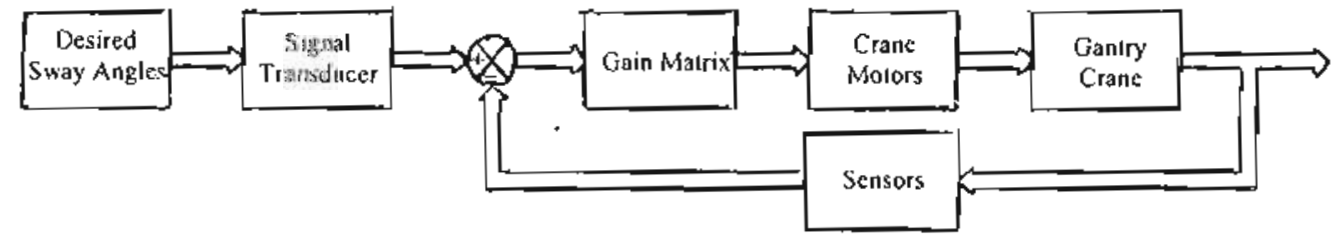

Fig. 6. Block Diagram of the Control Scheme.

$\Phi$

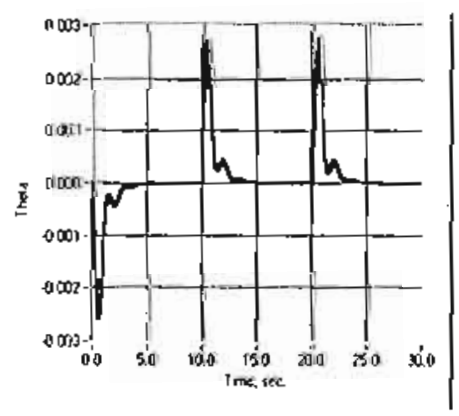

Fig. 7. Sway angle of $\theta_{l}$ with controi 


\section{NOMENCLATURE}

A gyotem effective inextia matrix

$A_{11}, A_{21}, A_{22}$ system submatrices,

$\dot{B}$ sygtem matrix,

$B_{1}, B_{2}$ gyatem submatricea,

$b_{1}, b_{2}, b_{1}, b_{t}$ gystem vectors,

e: subscript denctes the container coordinate

$\left[C_{2}\right],\left[C_{2 z}\right]$ system damping matrices,

¿ subscript denotes the direct coordinate in the d-g system of coordinates,

$E_{1}$ vector includes the ith motor steady state $\mathrm{Elux}$,

E matrix maps the motor effect to the mechanical part,

$E_{3}$ matrix represents the motors effect.

$F_{x}, F_{y}$ plane forceg acting on the girder and trolley $[N]$,

$F_{H}$ rope tension force [N],

$g$ gravitational acceleration [m/sec ${ }^{2}$,

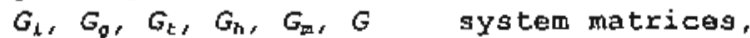

b gubscript denotes the hoisting,

If, Ir mass moment of inertia of the object in the axial and trangverse directions, [kg $\left.\mathrm{m}^{2}\right]$,

$I_{1}, I_{2}$ and $I_{3}$ mass moment of Inertia of the gixder motor, trolley motor and hoisting motor respectively $\left[\mathrm{Kg} \cdot \mathrm{m}^{2}\right]$.

$I_{\theta}=M i^{2}+I_{t} \quad$ aquivalent mag moment of inertia.

$I_{3,}, I_{1 d}, I_{1, d}$ etc. currents, and subgcriptg are defined as their

$j \quad j=(-1)^{0} 5$ locations [Amp.]

$\left[K_{2}\right],\left\langle K_{2 Z}\right\rfloor$ system matrices.

1 distance from the upper point of the unwound part of the rope to the mass center: of the load,

- subscript

$M$ suspended object mass [Kg] ,

$\mathbf{m}_{x}=M+m_{1}+m_{2}+\left(I_{1} / I_{1}{ }^{2}\right)$ equivalent mass [Kg],

$M_{\gamma}=H+m_{2}+\left(I_{2} / x_{2}{ }^{2}\right)$ equivalent mass [Kg],

$H_{1}=M+\left(I_{3} / r_{3}{ }^{2}\right)$ gquivalent mass [Kg],

$A_{1}$, and $m_{2} g i c d e r$ and trolley masses respectively [Kg],

$\left[\mathrm{M}_{2}\right],\left[\mathrm{M}_{11}\right],\left[\mathrm{H}_{22}\right]$ system mass matrices,

m: aubscript denotes the mechanical aystem

Q:siffness of the wire rope[N.w/rad]

$I_{1}$ radius of girder motor pinion [m],

$r_{2}$ radius of trolley rotox pinon [m],

Ij radius of drun [m].

r. subscript denotes the rotor.

$R_{1}$. resistance, the subscripte are to checked [Onm]

- subscript denotes the stator.

(T) transformation matrix

$t$ tine [gac],

t subscript denotes the trolley,

$\sigma$ vector denotes the input to the main system.

$\nabla_{1}$ vector denotes the input the ith aubsystem,

$U_{1 s}$ voltageg on the 1 th gtator [Volt].

u, v, crane cogrdination.

$x \quad x$-coordinate of the rope end [ $m$ ]

$x_{1}$ vector denotes the states of the ith motor,

$x$ system vector.

I $y^{-}$coordinate of rope end [r], gystem vectar,

$z$ - coordinate of rope end [m].

$z$ - coordinate of the object $[\mathrm{m}]$.

vector denotes the atate of the hole syatem,

$z_{n}$ vector denotes the state of the mechanical part. 


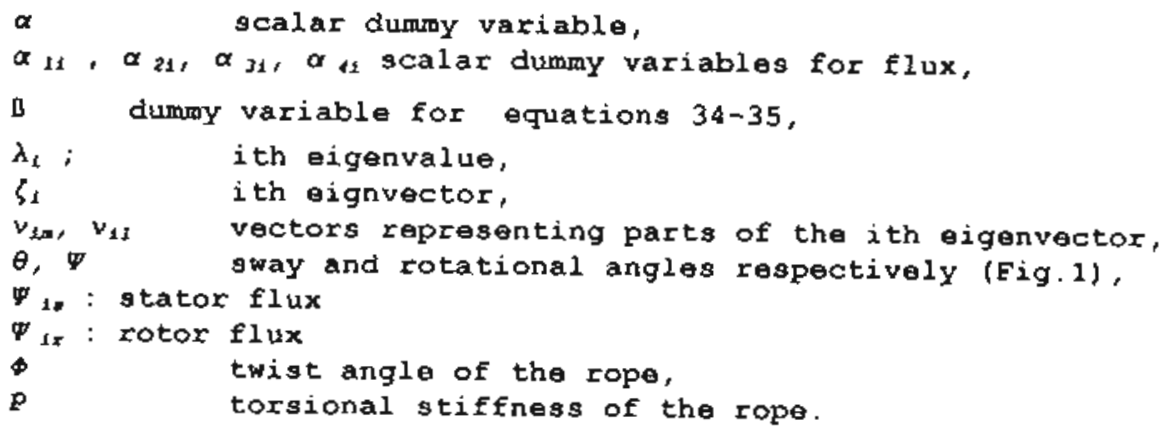

\section{APPENDIX A SXSTEM MATRICES}

$A(q)=\left[\begin{array}{ccccc}M_{u} & 0 & M \theta_{1} \sin \theta_{2} & M \sin \theta_{2} & M \theta_{1} \cos \theta_{2} \\ 0 & M_{v} & M \theta_{1} \cos \theta_{2} & M / \cos \theta_{2} & -M \theta_{1} \sin \theta_{2} \\ M \theta_{1} \sin \theta_{2} & M \theta_{1} \cos \theta_{2} & M_{1} & 0 & 0 \\ M l \sin \theta_{2} & M l \cos \theta_{2} & 0 & I_{\theta 1} & 0 \\ M l \theta_{1} \cos \theta_{2} & -M \theta_{1} \sin \theta_{2} & 0 & 0 & I_{\theta 2}\end{array}\right]$

$C(q, \dot{q})=M\left[\begin{array}{c}2 \dot{i} \dot{\theta}_{2} \theta_{1} \cos \theta_{2}+2 \dot{l} \dot{\theta}_{1} \sin \theta_{2}+2 l \dot{\theta}_{1} \dot{\theta}_{2} \cos \theta_{2}-l \dot{\theta}_{2}^{2} \theta_{1} \sin \theta_{2}-l \dot{\theta}_{1}^{2} \theta_{1} \sin \theta_{2} \\ -2 i \dot{\theta}_{2} \theta_{1} \sin \theta_{2}+2 l \dot{\theta}_{1} \cos \theta_{2}-2 l \dot{\theta}_{1} \dot{\theta}_{2} \sin \theta_{2}-l \dot{\theta}_{2}^{2} \theta_{1} \cos \theta_{2}-l \dot{\theta}_{1}^{2} \theta_{1} \cos \theta_{2} \\ l\left(\dot{\theta}_{2}^{2} \theta_{1}+\dot{\theta}_{1}^{2}\right) \\ 2 l i \dot{\theta}_{1}+l^{2} \dot{\theta}_{2}^{2} \theta_{1} \\ 2 i \dot{\theta}_{2} \theta_{1}{ }^{2}+\left(\left(2 M l^{2}-2 I_{1}+2 I_{2}\right) / M \dot{\theta}_{2} \dot{\theta}_{1} \theta_{1}\right.\end{array}\right]$

$J=\left[\begin{array}{cc}I_{\theta 1} & 0 \\ 0 & I_{\theta 1}\end{array}\right], D=\left[\begin{array}{cc}2 M i_{0} & 0 \\ 0 & 2 M i_{0} \theta_{10}^{2}\end{array}\right], K=\left[\begin{array}{cc}M g l_{0} & 0 \\ 0 & Q\end{array}\right]$,

$B=\left[\begin{array}{ccc}M_{0} \sin \theta_{20} & M_{0} \cos \theta_{20} & 0 \\ M_{0} \theta_{10} \cos \theta_{20} & -M_{0} \theta_{10} \sin \theta_{20} & 0\end{array}\right]$

$B_{1}=\left[\begin{array}{ccc}M_{u} & 0 & M \theta_{10} \sin \theta_{20} \\ 0 & M_{v} & M \theta_{10} \cos \theta_{20} \\ M \theta_{10} \sin \theta_{20} & M \theta_{10} \cos \theta_{20} & M_{i}\end{array}\right]$,

$F_{l}^{T}=\left[\begin{array}{lll}E_{r} X_{r}, & E_{r} X_{l}, & -E_{h} X_{h}\end{array}\right]$

$J_{1}=\left[\begin{array}{cc}M_{0} \sin \theta_{20} & M_{0} \theta_{10} \cos \theta_{20} \\ M_{0} \cos \theta_{20} & -M_{0} \theta_{10} \sin \theta_{20} \\ 0 & 0\end{array}\right], \quad D_{1}=\left[\begin{array}{cc}2 M i_{0} \sin \theta_{20} & 2 M_{0} \theta_{10} \cos \theta_{20} \\ 2 i_{0} \cos \theta_{20} & -2 M i_{0} \theta_{10} \sin \theta_{20} \\ 0 & 0\end{array}\right]$ 